\title{
Multivalent ligand-receptor-mediated interaction of small filled vesicles with a cellular membrane
}

\author{
Vladimir P. Zhdanov* \\ Division of Biological Physics, Department of Physics, Chalmers University of Technology, S-41296 Göteborg, Sweden \\ and Boreskov Institute of Catalysis, Russian Academy of Sciences, Novosibirsk 630090, Russia
}

(Received 23 March 2017; revised manuscript received 23 May 2017; published 17 July 2017)

\begin{abstract}
The ligand-receptor-mediated contacts of small sub-100-nm-sized lipid vesicles (or nanoparticles) with the cellular membrane are of interest in the contexts of cell-to-cell communication, endocytosis of membrane-coated virions, and drug (RNA) delivery. In all these cases, the interior of vesicles is filled by biologically relevant content. Despite the diversity of such systems, the corresponding ligand-receptor interaction possesses universal features. One of them is that the vesicle-membrane contacts can be accompanied by the redistribution of ligands and receptors between the contact and contact-free regions. In particular, the concentrations of ligands and receptors may become appreciably higher in the contact regions and their composition may there be different compared to that in the suspended state in the solution. A statistical model presented herein describes the corresponding distribution of various ligands and receptors and allows one to calculate the related change of the free energy with variation of the vesicle-engulfment extent. The results obtained are used to clarify the necessary conditions for the vesicle-assisted pathway of drug delivery.
\end{abstract}

DOI: 10.1103/PhysRevE.96.012408

\section{INTRODUCTION}

Small sub-100-nm-sized lipid vesicles (e.g., exosomes) play an important role in biology due to their ability to transport various content to and between cells. In particular, such vesicles participate in cell-to-cell communication by serving as key genetic, protein, and lipid carriers in a variety of biological processes related to the immune system, cancer, and neurodegenerative diseases [1]. Many viruses use a lipidmembrane envelope, which may be viewed as a vesicle, for their defense and facilitation of the entry into the host cells [2]. In applications, small vesicles or lipid nanoparticles (which are to some extent reminiscent of multilamellar vesicles) can potentially be employed as vehicles for drug (e.g., RNA) delivery [3-5]. In all these cases, the vesicles are filled and their interaction with a cellular membrane is typically mediated by pairs of ligands and receptors as schematically shown in Fig. 1.

Chemically, ligands and receptors are proteins and/or special lipids, and often these terms can be used interchangeably. On the scale of covalent bonds, each ligand-receptor interaction is usually relatively weak (from a few to $\sim 10 k_{\mathrm{B}} T$ ). Firm binding includes numerous ligand-receptor pairs and accordingly can be classified as multivalent (for the use of this term, see Ref. [6]; for advances in the corresponding experimental quantification techniques, see, e.g., Refs. [7,8]). Such multivalent interaction controls also adhesion between cells and may control adhesion of cells to a supported lipid bilayer, and it has long been studied experimentally and theoretically in the latter contexts (for the theory, see, e.g., reviews [9-13], recent articles [14-17], and references therein; many related theoretical studies are reviewed in Ref. [18]). In the corresponding kinetic models, the focus is customarily on the situations when the membranes contain one type of ligands and receptors, and their concentrations outside the contact region are usually considered to be constant.

\footnotetext{
*zhdanov@ catalysis.ru
}

In vivo, the cellular membrane and vesicles contain various receptors and ligands, respectively. During a contact of a filled vesicle with the cellular membrane (Fig. 1), the formation of ligand-receptor pairs occurs only in the contact area. This energetically favorable process may be accompanied by redistribution of ligands and receptors provided their diffusion is sufficiently fast, and their concentrations in the contact area may become higher. The specifics of the redistribution of receptors is that the increase of their concentration in the contact area does not change their concentration in the cellular membrane outside this area. Basically, the situation with the redistribution of receptors is similar to that in the case of cell adhesion (as already noticed, this case was earlier analyzed in many studies; in our context, we may first of all refer to the important works by Lipowsky et al. $[14,19,20])$. In a vesicle, in contrast, the increase of the concentration of ligands in the contact area may result in appreciable depletion of ligands outside this area (compared to the case when a vesicle is far from the cellular membrane). If a vesicle contains ligands of two or more types, other features are also possible. In particular, the increase of the concentration of ligands of one type in the contact area may result in appreciable enhancement of the number of ligands of another type in the no-contact area. Thus, the situation with the redistribution of ligands is similar to that in the case of cell adhesion only partly.

In the available kinetic models of receptor-mediated viral endocytosis [21-23] and related models [24-31] and simulations [32-37] of nanoparticle binding, the redistribution of receptors is usually taken into account while the redistribution of ligands is neglected. The latter is valid provided the ligand diffusion is negligible or if the ligands are of one type and their population is close to saturation. Such conditions are, however, not always met. The redistribution of ligands with explicit treatment of depletion of ligands in the no-contact area was analyzed by Smith and Seifert [38] in the context a vesicle adhering to a flat substrate and also was briefly addressed in the simulations by Schubertova et al. [33] in the context of nanoparticle uptake efficiency. Both these studies 

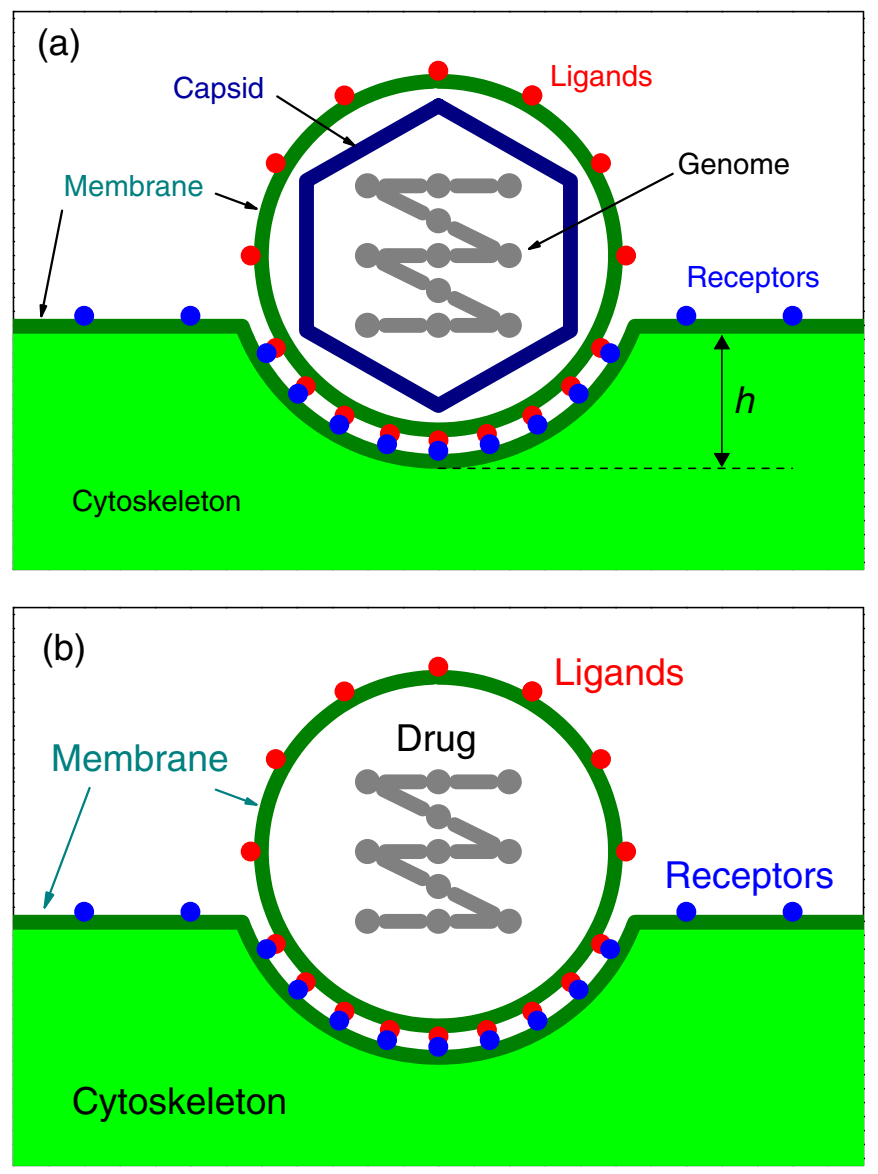

FIG. 1. Scheme of engulfment of (a) a membrane-coated virion and (b) vesicle into the host cell due to the ligand-receptor interaction. $h$ is the engulfment depth. Ligands and receptors are shown by small filled circles, irrespective of their type.

were focused on the case of one type of ligands and one type of receptors.

Herein, our goal is to scrutinize analytically and by related numerical calculations the features of multivalent interaction between small vesicles and the cellular membrane and to clarify their manifestation in the context of endocytosis of membrane-coated virions and vesicle-mediated drug delivery. Compared to the earlier studies aimed at the viral endocytosis and nanoparticle-mediated drug delivery, we take ligands and receptors of various types into account, pay special attention to the changes of the receptor and ligand concentrations in the contact area and to the changes of the ligand concentrations outside this area, and illustrate in detail the corresponding ligand distribution and the dependence of the vesicle free energy on the vesicle-engulfment extent.

\section{METHODS}

\section{A. General remarks}

In our analysis, a vesicle is considered to contain ligands of various types. The ligands are assumed to be flexible, and their size (a few nanometers) is much smaller than the vesicle size $(\sim 100 \mathrm{~nm})$. The cellular membrane contains various flexible receptors, and their size (a few nanometers) is assumed to be much smaller than the vesicle size as well. The formation and rupture of ligand-receptor bonds in the contact area between a vesicle and the cellular membrane and their redistribution are considered to be rapid, because (i) the bonds are weak and (ii) on the length scale of small vesicles $(\sim 100 \mathrm{~nm})$ the diffusion of ligands and receptors in the membranes is often relatively fast.

Concerning the diffusion, one should take into account that its rate is limited by reconfiguration of lipids (in fact, some of the lipids may serve as receptors). In particular, the diffusion coefficient of ligands and receptors is expected to be lower that the diffusion coefficient of lipids by a factor of $3-5$. The latter diffusion coefficient is often in the range from 1 to $5 \mu \mathrm{m}^{2} / \mathrm{s}$ (see, e.g., Refs. $[39,40]$ ). In addition to various lipids, cell membranes include proteins and cholesterol, which may slow down diffusion of ligands and receptors, but this effect is not necessarily dramatic [41]. Taken together, these data indicate that the diffusion of ligands and receptors is indeed often (but not always) relatively fast, and this is an acceptable starting point. For this reason, we consider that at any given contact area the distributions of ligands and receptors is close to equilibrium and can be described by employing the conventional prescriptions of statistical mechanics.

To calculate the distributions of ligands and receptors and to show the contribution of this factor to the thermodynamic driving force for wrapping of a filled vesicle by the cellular membrane, one should determine and minimize a suitable thermodynamic potential. In the case under consideration, the number of ligands in a vesicle is fixed, and the number of receptors in the cellular membrane is fixed as well. Thus, in principle, one can use here the canonical distribution and operate with the the Helmholtz free energy, $F$ (Eq. (31.3) in Ref. [42]). The latter number is, however, very large, and accordingly the cellular membrane can be considered as an unlimited reservoir of receptors with the fixed chemical potential defined by the receptor concentration outside the vesicle-membrane contact region. Under such circumstances, the receptors located in the contact region can be described by employing the grand canonical distribution and one can operate with the omega-potential, $\Omega$ (Eq. (35.3) in Ref. [42]). The use of the latter distribution for receptors appreciably simplifies the analysis. In particular, the analysis of the ligand-receptor interaction can be fully focused on a vesicle, including its two parts contacting and not contacting the cellular membrane while the receptors located outside the contact area can be excluded from the explicit treatment. Following this line, we below first employ the grand canonical distribution to calculate the effective energy of the interaction of a ligand with receptors. Then, this energy is used to determine the contribution of the ligand-receptor interaction to the vesicle Helmholtz free energy and to calculate the ligand distribution.

In general, the free energy and omega-potential are expressed, respectively, via the canonical and grand canonical partition functions, which take all possible arrangements of particles into account [42]. To simplify the calculation of the partition functions, we employ a coarse-grained model based on the conventional lattice-gas approximation $[43,44]$. 
In particular, we introduce the maximum possible number of ligands in a vesicle, $N$, and treat a vesicle as a lattice containing $N$ sites each with the area $a=4 \pi R^{2} / N$, where $R$ is the vesicle radius. In turn, the cellular membrane is also treated as a lattice composed of the sites for receptors with its own maximum number of sites. For simplicity, the area of the latter sites is considered to be $a$ as well. Focusing on the formation of the ligand-receptor bonds, we neglect the lateral ligand-ligand and receptor-receptor interactions. In principle, such interactions can be taken into account in various approximations $[43,44]$, but this is beyond our present goals.

The general approach outlined above is, of course, not original. The lattice models were, e.g., earlier widely used in various fields of chemical physics ranging from protein folding [45] and adsorption [46] to heterogeneous catalytic reactions [44]. In the context of cell adhesion, this model was employed in the seminal study by Weikl and Lipowsky [19] and its extensions [14,20] (see also review [10]). For attachment of vesicles to a supported lipid model, the model was used in the already-mentioned study by Smith and Seifert [38]. Our analysis is inevitably partly overlapping with the latter studies and simultaneously, as discussed below, contains ingredients and results related to viral endocytosis and drug delivery.

\section{B. Interaction of a single ligand with receptors}

During a contact of a vesicle with the cellular membrane, a ligand located in one of the sites in the contact area on the vesicle side is able to form a bond with a receptor situated in the corresponding nearest-neighbor (nn) site on the cellmembrane side. The latter site can be vacant or occupied by one of the receptors, which may form a bond with a ligand. To describe the effective interaction of a ligand, situated in the contact area, with receptors, we use the grand canonical partition function for the cell-membrane site which is nn to the site occupied by a ligand of type $i$,

$$
\Sigma_{1}=1+\sum_{j} \exp \left(\mu_{j} / k_{\mathrm{B}} T\right)+\sum_{j} \exp \left[\left(\mu_{j}-\epsilon_{i j}\right) / k_{\mathrm{B}} T\right],
$$

where $\epsilon_{i j}$ is the bond energies of the pairs including a ligand of this type and a receptor of type $j$, and $\mu_{j}$ is the receptor chemical potential. The three terms on the right-hand part of this expression correspond to the situations when the site is (i) vacant, (ii) occupied by a receptor which does not form a bond with a ligand, and (ii) occupied by a receptor which forms a bond with a ligand, respectively. The corresponding $\Omega$ potential is represented via this partition function as

$$
\begin{aligned}
\Omega_{1}= & -k_{\mathrm{B}} T \ln \left\{1+\sum_{j} \exp \left(\mu_{j} / k_{\mathrm{B}} T\right)\right. \\
& \left.+\sum_{j} \exp \left[\left(\mu_{j}-\epsilon_{i j}\right) / k_{\mathrm{B}} T\right]\right\} .
\end{aligned}
$$

If a site in the contact area on the vesicle side is not occupied by a ligand, then the grand canonical partition function and $\Omega$ potential for the $n n$ site on the cell-membrane side are defined as

$$
\begin{gathered}
\Sigma_{0}=1+\sum_{j} \exp \left(\mu_{j} / k_{\mathrm{B}} T\right), \\
\Omega_{0}=-k_{\mathrm{B}} T \ln \left[1+\sum_{j} \exp \left(\mu_{j} / k_{\mathrm{B}} T\right)\right] .
\end{gathered}
$$

The change of the $\Omega$ potential, related to the bond formation and given by the difference of Eqs. (2) and (4), can be identified with the effective interaction of a ligand with receptors,

$$
\begin{aligned}
e_{i}= & -k_{\mathrm{B}} T \ln \\
& \times\left\{\frac{1+\sum_{j} \exp \left(\mu_{j} / k_{\mathrm{B}} T\right)+\sum_{j} \exp \left[\left(\mu_{j}-\epsilon_{i j}\right) / k_{\mathrm{B}} T\right]}{1+\sum_{j} \exp \left(\mu_{j} / k_{\mathrm{B}} T\right)}\right\} .
\end{aligned}
$$

As already noticed in Sec. II A, the chemical potential of receptors is determined by their concentration outside the contact region. In the framework of the lattice-gas model, the well-known exact expression for this potential for receptors of type $j$ is

$$
\mu_{j}=k_{\mathrm{B}} T \ln \left[\Theta_{j} /\left(1-\Theta_{\mathrm{t}}\right)\right],
$$

where $\Theta_{j}<1$ is the normalized population (i.e., the population per site) of the receptors of interest, and $\Theta_{t}=\sum_{j} \Theta_{j}$ is the total normalized receptor population. This expression takes saturation of the sites into account. If the total coverage is low, $\Theta_{\mathrm{t}} \ll 1$, and the saturation is negligible, it is reduced to

$$
\mu_{j} \simeq k_{\mathrm{B}} T \ln \Theta_{j}
$$

In vivo, the situation may or may not be far from saturation depending on the extent of the membrane crowding.

Substituting Eq. (6) into Eq. (5) yields the following general expression for effective interaction of a ligand with receptors:

$$
e_{i}=-k_{\mathrm{B}} T \ln \left[1+\sum_{j} \Theta_{j} \exp \left(-\epsilon_{i j} / k_{\mathrm{B}} T\right)\right] \text {. }
$$

This interaction can be used to describe the distribution of receptors between the contact and contact-free areas of a vesicle.

Comparing the derivation of our Eqs. (1)-(8) with the earlier treatments $[14,19,20]$ mentioned in Sec. II A, one can notice four aspects. (i) References [14,19,20] are focused on cell adhesion, and the ligand-ligand and receptor-receptor distances are there considered to be appreciably larger than the site size or, in other words, the ligand and receptor concentrations are typically assumed to be low, and the analysis takes thermally excited shape fluctuations of the membrane into account. We are interested in endocytosis of virions and drug delivery where the ligand concentration is typically appreciable, and the receptor concentration may be appreciable as well (the corresponding vesicles or lipid nanoparticles may contact various receptors), and accordingly we take saturation into account but do not pay attention to the membrane roughness. The role of the latter factor decreases with increasing local concentration of bonds [20]. In a filled vesicle, the roughness can be reduced also due 
to the content inside. (ii) Treatments $[14,19,20]$ imply that the nn ligand and receptor form a bond. In our model, the nn ligand and receptor can be bound and unbound. This aspect is important if one wants to identify the difference in the formalism. Practically, this difference is negligible at low receptor concentration (outside the contact area) but may be significant at appreciable receptor concentration. (iii) To derive the final compact expression for the effective ligand-receptor interaction [Eq. (34) in Ref. [20] (or Eq. (6) in Ref. [14])], the authors of Refs. [14,19,20] not only consider that the ligand and receptor concentrations are low but also imply that the pair ligand-receptor interaction energy, $U>0$ (or $-\epsilon_{i j}$ in our designations) is sufficiently small so that $a^{4}[R][L] \exp \left(U / k_{\mathrm{B}} T\right) \ll 1$ (see, e.g., Eqs. (32) and the text above in Ref. [20]), where $[R]$ and $[L]$ are the receptor and ligand concentrations, and $a^{2}$ (or $a$ in our designations) is the site area. In this limit, the effective interaction calculated per unit area is given by (see Eq. (34) in Ref. [20] or Eq. (6) in Ref. [14])

$$
U_{\mathrm{ef}}=k_{\mathrm{B}} T[R][L] a^{2} \exp \left(U / k_{\mathrm{B}} T\right) .
$$

The corresponding effective interaction per ligand is

$$
e=k_{\mathrm{B}} T[R] a^{2} \exp \left(U / k_{\mathrm{B}} T\right)=k_{\mathrm{B}} T \Theta \exp \left(U / k_{\mathrm{B}} T\right),
$$

where $\Theta=[R] a^{2}$ is the receptor population per site. Except the sign (which depends on the choice), the latter expression is identical to that predicted by Eq. (8) in the case of receptors of one type provided $\Theta_{j} \exp \left(-\epsilon_{i j} / k_{\mathrm{B}} T\right) \ll 1$ [under this condition, one can expand Eq. (8) and keep the first linear term]. In our analysis above and calculations presented below (in Sec. III), we do not use the latter condition because this limit does not seem to be suitable in the contexts of endocytosis of virions and drug delivery (see the corresponding remark in Sec. IV). (iv) In Ref. [20], one can also find a more general expression [Eq. (25) in combination with Eqs. (29)-(31)] for the effective adhesion potential at arbitrary ligand and receptor concentrations. The latter expression is far from compact. In addition, using the conventional expression for the chemical potential [Eq. (6)], one can easily verify that at appreciable receptor concentration (outside the contact area) it is not fully identical to Eq. (8). The physics behind the difference has already explained in item (ii) above.

The effective ligand-receptor interaction was also calculated in Ref. [38]. The corresponding expression (Eq. (8) in Ref. [38]) is, however, not explicit, because it contains variables $\left(N_{b}\right.$ and $\left.N_{f}\right)$ that must be calculated from other equations. For this reason, its comparison with Eq. (8) is not straightforward.

\section{Free energy and distribution of ligands}

The number of ligands in a vesicle is fixed, and as already noticed in Sec. II A, we can use the canonical distribution and operate with the the Helmholtz free energy to determine the driving force for engulfment of a filled vesicle into the host cell and the ligand distribution between the contact and contact-free regions. In particular, we operate with the change of free energy, $\Delta F$, during a contact of a vesicle with the cellular membrane. This value is represented as

$$
\Delta F=\Delta F_{\mathrm{lr}}+\Delta E_{\mathrm{b}}+\Delta E_{\mathrm{c}},
$$

where $\Delta F_{\text {lr }}<0$ is the contribution related to the ligandreceptor interaction, $\Delta E_{\mathrm{b}}>0$ is the change of the membrane bending energy, and $\Delta E_{\mathrm{c}}>0$ is the change of the deformation energy of the cytoskeleton or, more specifically, actin filaments. Our present study is focused on the analysis of $\Delta F_{\mathrm{lr}}$. The two other terms, $\Delta E_{\mathrm{b}}$ and $\Delta E_{\mathrm{c}}$, are briefly discussed for comparison (Sec. IV). The term we are interested in is represented as

$$
\Delta F_{\mathrm{lr}}=F_{1}-F_{1}^{\star},
$$

where $F_{1}$ is the free energy of ligands (including their interaction with receptors) during the contact a vesicle with the cellular membrane, and $F_{1}^{\star}$ is the corresponding reference free energy of ligands calculated in the no-contact case.

If a vesicle is in the suspended (no-contact) state, the free energy of ligands is given by

$$
F_{1}^{\star}=-k_{\mathrm{B}} T \ln \left(\frac{N !}{\left(N-n_{\mathrm{t}}\right) ! \Pi_{i} n_{i} !}\right),
$$

where $n_{i}$ is the population (number) of ligands of type $i$, and $n_{\mathrm{t}}=\sum_{i} n_{i}$ is the total ligand population. This expression for $F_{1}^{\star}$ is valid for arbitrary populations $n_{i}$. Usually, the populations of interest are appreciable $\left(n_{i} \gg 1\right)$. In this case, the use of Stirling's formula $\ln n ! \simeq n \ln (n / e)$ allows one to rewrite Eq. (13) as

$$
F_{1}^{\star}=k_{\mathrm{B}} T N\left[\sum_{i} \theta_{i} \ln \theta_{i}+\left(1-\theta_{\mathrm{t}}\right) \ln \left(1-\theta_{\mathrm{t}}\right)\right],
$$

where $\theta_{i}=n_{i} / N$ and $\theta_{\mathrm{t}}=\sum_{i} \theta_{i}$ are the normalized specific and total populations, i.e., populations per site.

For a vesicle contacting the cellular membrane, the free energy of ligands including their interaction with receptors is represented as a sum of two contributions,

$$
F_{1}=F_{1}^{\circ}+F_{1}^{*},
$$

taking, respectively, the contact-free and contact areas into account. The former contribution is expressed by analogy with Eq. (14) as

$$
F_{1}^{\circ}=k_{\mathrm{B}} T N_{\circ}\left[\sum_{i} \theta_{i}^{\circ} \ln \theta_{i}^{\circ}+\left(1-\theta_{\mathrm{t}}^{\circ}\right) \ln \left(1-\theta_{\mathrm{t}}^{\circ}\right)\right],
$$

where $\theta_{i}^{\circ}=n_{i}^{\circ} / N_{\circ}$ and $\theta_{\mathrm{t}}^{\circ}=\sum_{i} \theta_{i}^{\circ}$ are the normalized partial and total ligand populations of sites in the contact-free area, and $N_{\circ}$ is the number of sites in this area. The latter contribution contains in addition the term taking the effective ligand-receptor interaction into account and is given by

$$
F_{1}^{*}=N_{*}\left\{\sum_{i} e_{i} \theta_{i}^{*}+k_{\mathrm{B}} T\left[\sum_{i} \theta_{i}^{*} \ln \theta_{i}^{*}+\left(1-\theta_{\mathrm{t}}^{*}\right) \ln \left(1-\theta_{\mathrm{t}}^{*}\right)\right]\right\} \text {, }
$$

where $\theta_{i}^{*}=n_{i}^{*} / N_{*}$ and $\theta_{\mathrm{t}}^{*}=\sum_{i} \theta_{i}^{*}$ are the normalized partial and total ligand populations of sites in the contact area, and $N_{*}$ is the number of sites in this area.

The sum of the numbers of sites on the two areas of a vesicle is equal to the total number of sites,

$$
N_{\circ}+N_{*}=N \text {. }
$$


In addition, there is balance relation for the ligand populations introduced above,

$$
\theta_{i}^{\circ} N_{\circ}+\theta_{i}^{*} N_{*}=N \theta_{i},
$$

where $\theta_{i}=n_{i} / N$ is the average population of sites by ligand $i$ [mathematically, this definition is equivalent to that used for $\theta_{i}$ in Eq. (14)].

The redistribution of ligands between the two areas results in equilibrium described by the condition

$$
\mu_{i}^{\circ}=\mu_{i}^{*},
$$

where

$$
\begin{aligned}
& \mu_{i}^{\circ}=\frac{1}{N_{\circ}} \frac{\partial F_{\circ}}{\partial \theta_{i}^{\circ}}=k_{\mathrm{B}} T \ln \left(\frac{\theta_{i}^{\circ}}{1-\theta_{\mathrm{t}}^{\circ}}\right) \text { and } \\
& \mu_{i}^{*}=\frac{1}{N_{*}} \frac{\partial F_{*}}{\partial \theta_{i}^{*}}=e_{i}+k_{\mathrm{B}} T \ln \left(\frac{\theta_{i}^{*}}{1-\theta_{\mathrm{t}}^{*}}\right)
\end{aligned}
$$

are the corresponding chemical potentials. Mathematically, Eq. (20) is direct consequence of minimization of the free energy with respect to ligand distribution under fixed contact geometry.

Taken together, Eqs. (18)-(22) allow one to describe the distribution of ligands between the contact-free and contact areas. Then, one can calculate the contribution of the ligand-receptor interaction into the change of the free energy [Eq. (12)].

\section{RESULTS OF CALCULATIONS}

The cellular membranes contain a lot of different receptors. The membranes of vesicles formed in vivo contain a lot of various ligands. In drug-delivery applications, the membranes of vesicles may contain various ligands as well. For illustration of general trends, we consider generic situations with receptors of two types and ligands of two types and use the values of the pair ligand-receptor interactions from the practically relevant range (see the Introduction). The normalized populations of specific receptors outside the contact region, $\Theta_{j}$, are chosen in our examples in the range from 0 to 0.2 . Although these populations are relatively low, the corresponding local populations in the contact region may often be close to saturation (see below). During viral endocytosis and in drug-delivery applications, the normalized ligand populations may be appreciable or even close to saturation. Below, these populations are selected to be 0.2 or 0.4 in the no-contact case, so that the total ligand populations are 0.4 and 0.8 , respectively.

Using Eq. (8), we have first calculated (Fig. 2) the effective energy of interaction of a ligand of one of the types (e.g., of type 1), $e_{1}$, with receptors for four sets of the pair ligand-receptor interactions: (i) $\epsilon_{11}=\epsilon_{12}=-3 k_{\mathrm{B}} T$, (ii) $\epsilon_{11}=-3 k_{\mathrm{B}} T$ and $\epsilon_{12}=-6 k_{\mathrm{B}} T$, (iii) $\epsilon_{11}=-6 k_{\mathrm{B}} T$ and $\epsilon_{12}=-3 k_{\mathrm{B}} T$, and (iv) $\epsilon_{11}=\epsilon_{12}=-6 k_{\mathrm{B}} T$. For each set of these interactions, $e_{1}$ is calculated for four normalized populations of receptors of type $1, \Theta_{1}=0.05,0.1,0.15$, and 0.2 , as a function of the normalized population of receptors of type 2 . In case (i), both interactions, $\epsilon_{11}$ and $\epsilon_{12}$, are relatively weak, and the dependence of the effective interaction, $f_{1}$, on $\Theta_{1}$ and $\Theta_{2}$ is weak as well. In addition, this dependence is nearly linear. In general, however, the dependence of $e_{1}$ on the receptor populations is not additive and accordingly nonlinear. This is illustrated, e.g., by

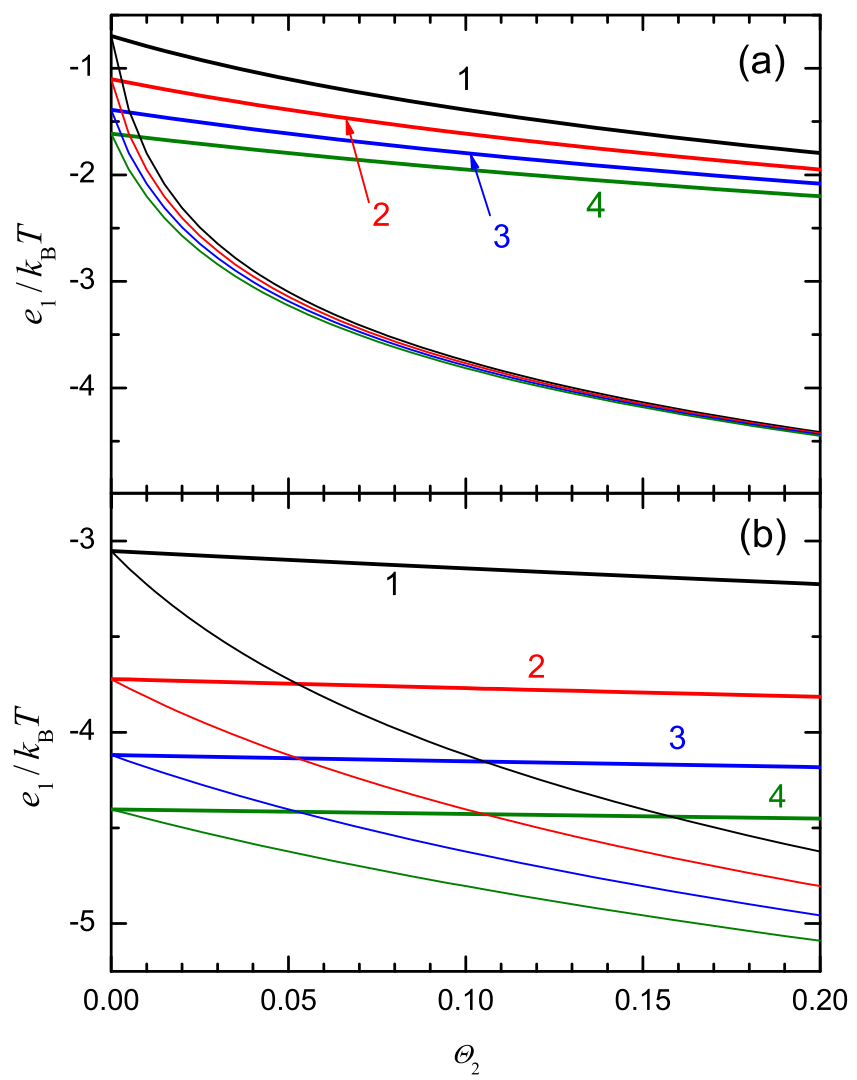

FIG. 2. Effective energy of the interaction of a ligand of type 1 with receptors as a function of normalized concentration (outside the contact region) of receptors of type 2 for four sets of the ligand-receptor interactions: (a) $\epsilon_{11}=\epsilon_{12}=-3 k_{\mathrm{B}} T$ (thick lines) and $\epsilon_{11}=-3 k_{\mathrm{B}} T$ and $\epsilon_{12}=-6 k_{\mathrm{B}} T$ (thin lines); and (b) $\epsilon_{11}=-6 k_{\mathrm{B}} T$ and $\epsilon_{12}=-3 k_{\mathrm{B}} T$ (thick lines) and $\epsilon_{11}=\epsilon_{12}=-6 k_{\mathrm{B}} T$ (thin lines). For each set of the ligand-receptor interactions, the results are presented for four normalized concentrations (outside the contact region) of receptors of type $1, \Theta_{1}=0.05,0.1,0.15$, and 0.2 (for two sets of the interactions, the corresponding lines are marked by numbers 1-4; for the other sets, the lines can be identified taking into account that with increasing $\Theta_{1}$ the absolute value of the interaction becomes larger).

cases (ii)-(iv). In case (ii), for example, $\epsilon_{11}$ is relatively small while $\epsilon_{12}$ is large, and the dependence of $e_{1}$ on $\Theta_{1}$ and $\Theta_{2}$ is accordingly weak and strong, respectively. Case (iii) is inverse to case (ii). In case (iv), $e_{1}$ depends appreciably on both receptor populations in a nonlinear fashion.

Employing Eqs. (18)-(22), we then scrutinized the distribution of ligands between the contact-free and contact areas. For example, the corresponding results or, more specifically, the normalized ligand populations of the contact and contactfree areas, $\theta_{1}^{*} N_{*} / N, \theta_{2}^{*} N_{*} / N, \theta_{1}^{\circ} N_{\circ} / N$, and $\theta_{1}^{\circ} N_{\circ} / N$, are presented in Fig. 3(a) as a function of the fraction of the sites forming the contact area, $N_{*} / N$, for three sets of effective ligand-receptor interactions: (i) $e_{1}=e_{2}=-3 k_{\mathrm{B}} T$, (ii) $e_{1}=-3 k_{\mathrm{B}} T$ and $e_{2}=-6 k_{\mathrm{B}} T$, and (iii) $e_{1}=e_{2}=$ $-6 k_{\mathrm{B}} T$. The average population of sites were fixed as $\theta_{1}=\theta_{2}=0.2$. In all three cases, with increasing $N_{*} / N$, the normalized populations of the contact area are seen to increase in such a manner that the population of ligands at 
(a)

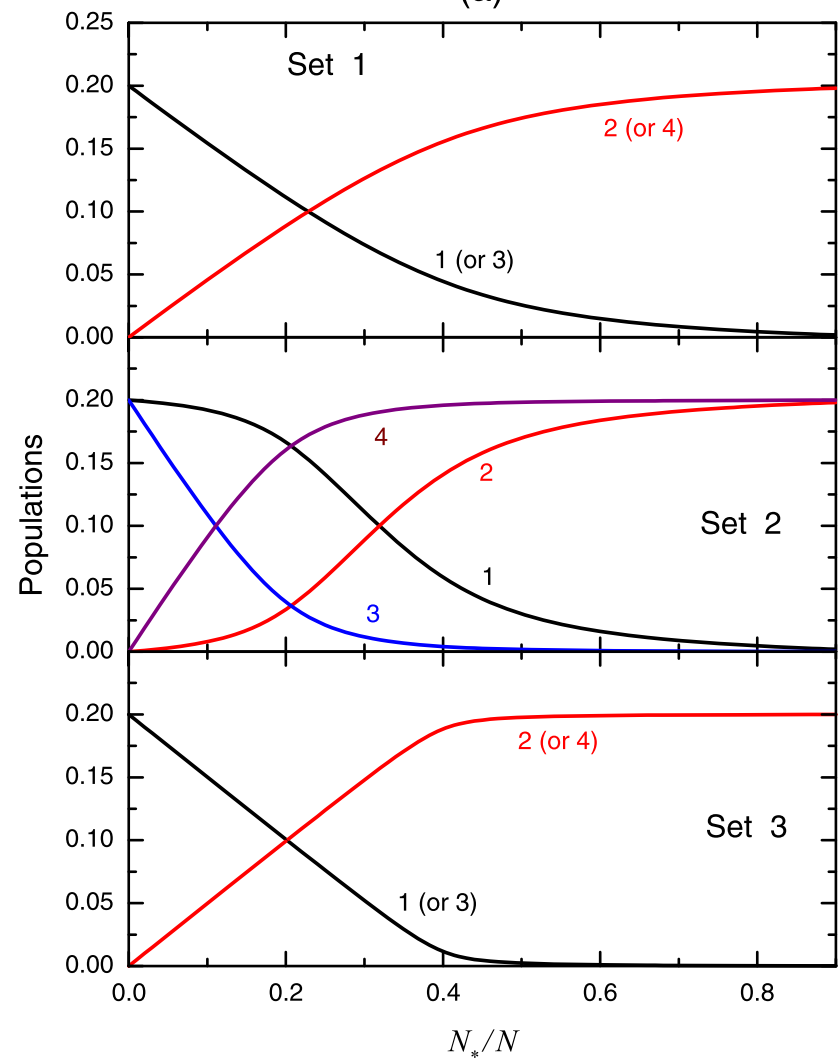

(b)

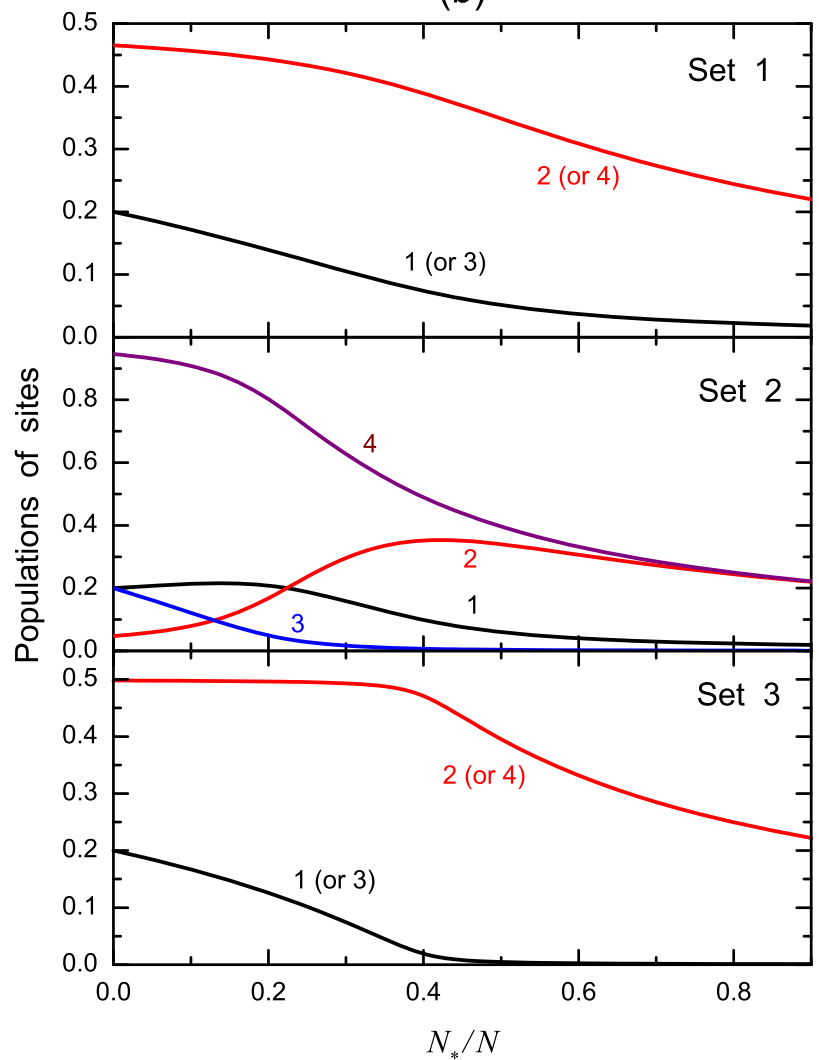

FIG. 3. Distribution of ligands between the contact-free and contact areas for $\theta_{1}=\theta_{2}=0.2$. Panel (a) shows the populations of ligands in these areas normalized to $N, \theta_{1}^{\circ} N_{\circ} / N$ (line 1), $\theta_{1}^{*} N_{*} / N$ (line 2), $\theta_{2}^{\circ} N_{\circ} / N$ (line 3), $\theta_{2}^{*} N_{*} / N$ (line 4), as a function of the fraction of the sites forming the contact area, $N_{*} / N$, for three sets of effective ligand-receptor interactions including (1) $e_{1}=e_{2}=-3 k_{\mathrm{B}} T$, (2) $e_{1}=-3 k_{\mathrm{B}} T$ and $e_{2}=-6 k_{\mathrm{B}} T$, and (3) $e_{1}=e_{2}=-6 k_{\mathrm{B}} T$. Panel (b) exhibits the corresponding populations of sites, $\theta_{1}^{\circ}$ (line 1), $\theta_{1}^{*}$ (line 2), $\theta_{2}^{\circ}$ (line 3 ), and $\theta_{2}^{*}$ (line 4).

the contact area is close to maximum. If the contact area is small $\left(N_{*} / N<\theta_{1}+\theta_{2}=0.4\right)$, a part of ligands cover almost completely this area and the other ligands are located beyond. If the contact area is appreciable $\left(N_{*} / N>\theta_{1}+\theta_{2}=0.4\right)$, the majority of the ligands are located in this area. As expected, these features of the ligand distribution are more pronounced with increasing effective ligand-receptor interactions. If the effective ligand-receptor interactions are equal, $e_{1}=e_{2}$ [cases (i) and (iii)], the normalized populations of the contact area, $\theta_{1}^{*} N_{*} / N$ and $\theta_{2}^{*} N_{*} / N$, increase synchronously. For $e_{1}=$ $-3 k_{\mathrm{B}} T$ and $e_{2}=-6 k_{\mathrm{B}} T$ [case (ii)], the contact area is first (at $N_{*} / N \leqslant \theta_{2}=0.2$ ) populated primarily by ligands of type 2 , because the effective interaction of these ligand with receptors is stronger. Then (at $N_{*} / N>\theta_{2}=0.2$ ), the contact area is populated by ligands of both types. All these features of the distribution of ligands can also be tracked in Fig. 3(b), showing the corresponding populations of sites, $\theta_{1}^{*}, \theta_{2}^{*}, \theta_{1}^{\circ}$, and $\theta_{1}^{\circ}$, in the contact and contact-free areas.

With the parameters given in the paragraph above, we have also calculated the change of the free energy of a vesicle, $\Delta F_{\text {lr }}$ [Eq. (12)], as a function of the fraction of contact sites, $N_{*} / N$ (Fig. 4). For all the sets of effective ligand-receptor interactions, with increasing $N_{*} / N$, the absolute value of this contribution first (at $N_{*} / N \leqslant \theta_{1}+\theta_{2}=0.4$ ) rapidly increases in a linear fashion and then (at $N_{*} / N>\theta_{1}+\theta_{2}=0.4$ ) its dependence on $N_{*} / N$ becomes weaker. These features of the dependence of $\Delta F_{1 \mathrm{lr}}$ on $N_{*} / N$ are directly related with the redistribution of ligands in favor of the contact area.

The results exhibited in Figs. 3 and 4 were obtained for $\theta_{1}=\theta_{2}=0.2$. The results for $\theta_{1}=\theta_{2}=0.4$ are qualitatively

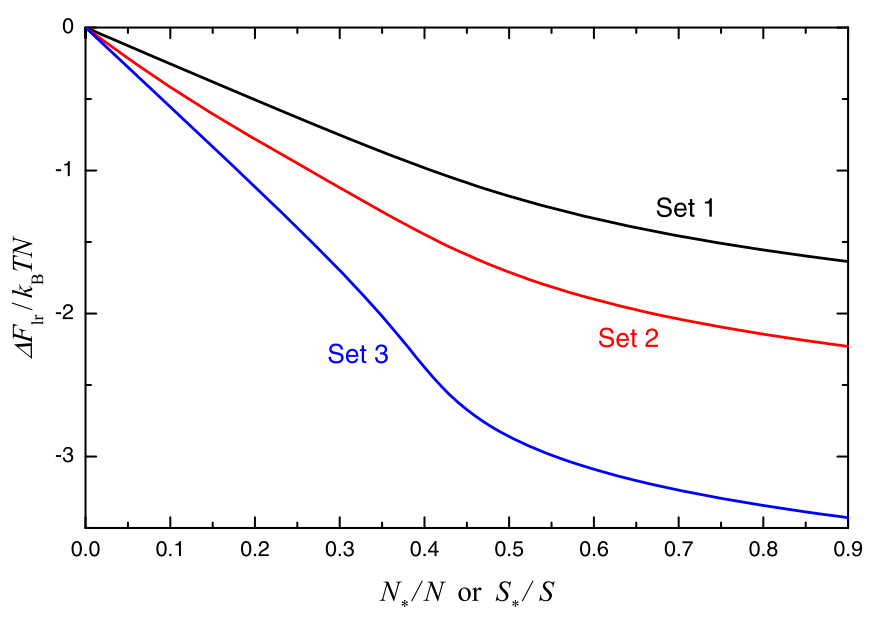

FIG. 4. Contribution of the ligand-receptor interaction to the change of the free energy of a vesicle [Eq. (12)] as a function of the fraction of the sites forming the contact area (or the fraction of contact area) for $\theta_{1}=\theta_{2}=0.2$ and three sets of effective ligand-receptor interactions (as in Fig. 3). 
(a)

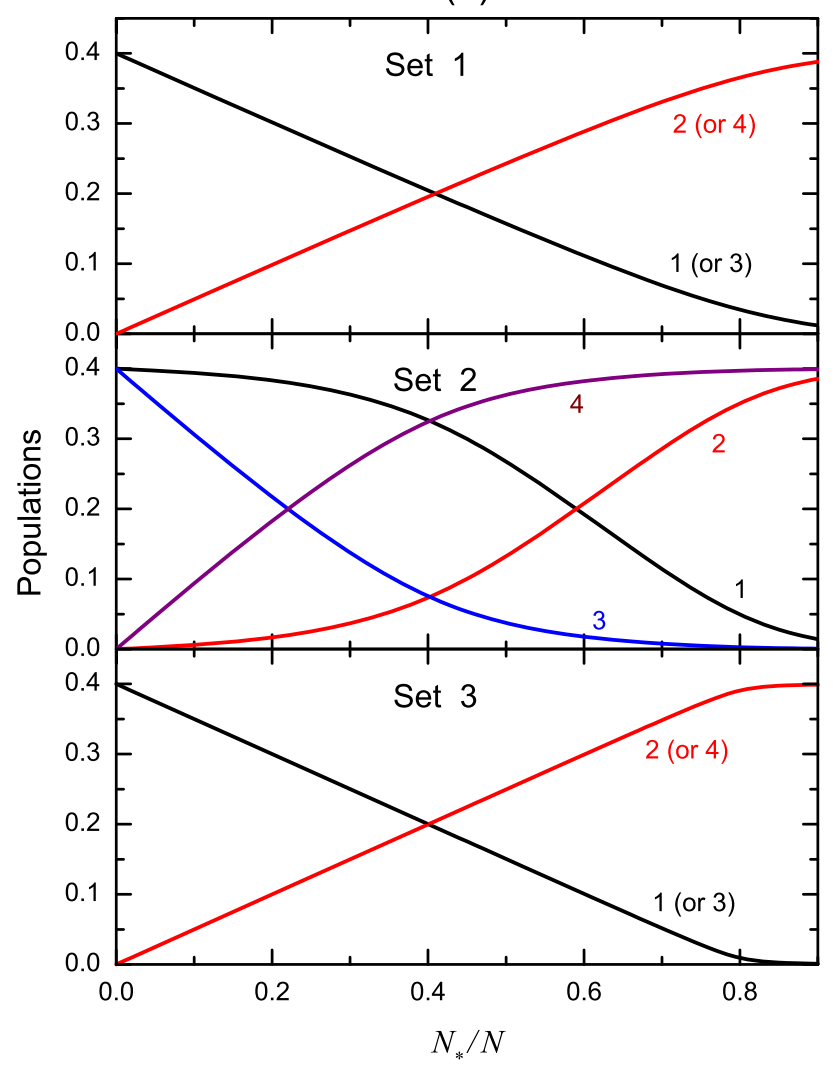

(b)

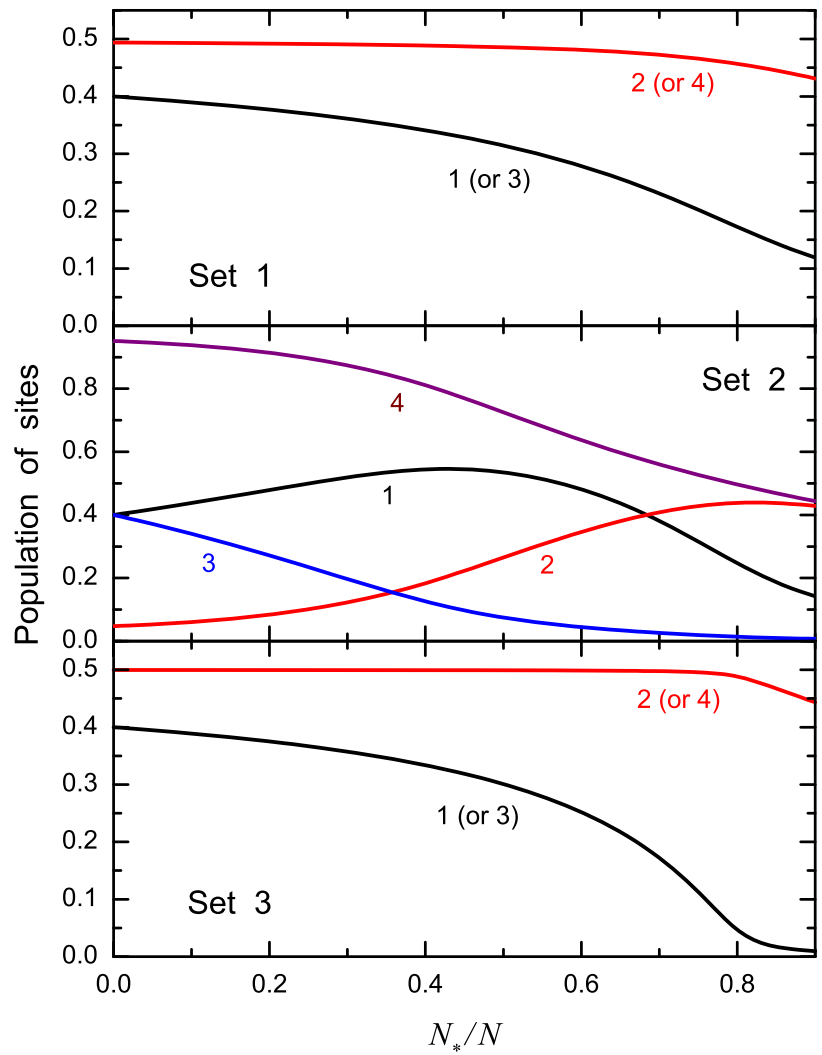

FIG. 5. As described in the caption of Fig. 3, except for $\theta_{1}=\theta_{2}=0.4$.

similar, except that the main features observed with increasing $N_{*} / N$ are shifted to the right (Figs. 5 and 6).

The results presented in Figs. 3-6 are shown as a function of the fraction of the sites forming the contact area, $N_{*} / N$. This fraction is equal to the fraction of the contact area, i.e., to the ratio of the contact area to the total vesicle area, $S_{*} / S$. If during a contact with the cellular membrane of a filled vesicle

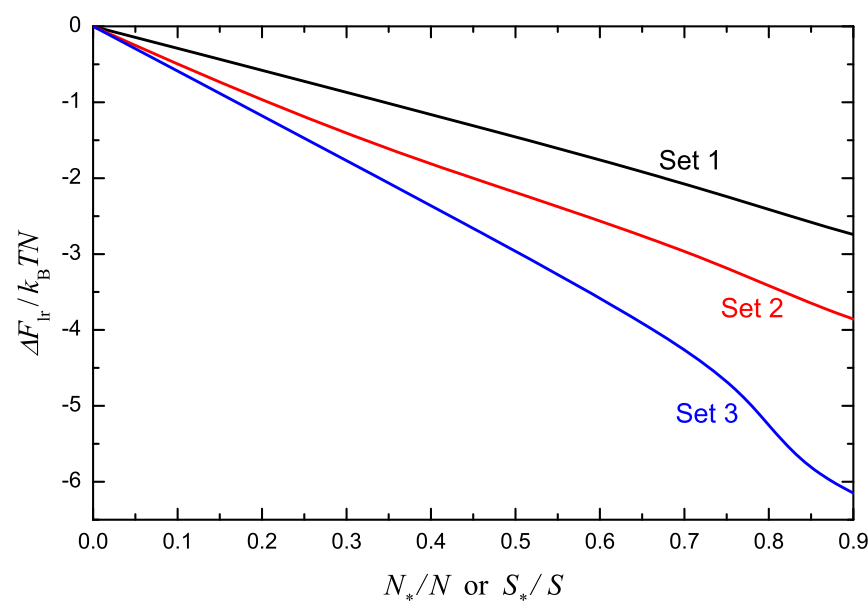

FIG. 6. Contribution of the ligand-receptor interaction to the change of the free energy of a vesicle [Eq. (12)] as a function of the fraction of the sites forming the contact area (or the fraction of contact area) for $\theta_{1}=\theta_{2}=0.4$ and three sets of effective ligand-receptor interactions (as described in the caption of Fig. 5). remains to be nearly spherically shaped (as, e.g., in Fig. 1), the contact area can be expressed via the engulfment depth, $h$, as $S_{*}=2 \pi h R$, and one has $N_{*} / N=S_{*} / S=h / 2 R$. These relations make it possible to operate by $S_{*} / S$ or $h / 2 R$ instead of $N_{*} / N$, i.e., these ratios can be used interchangeably.

\section{CONCLUSION}

Ligand-receptor-mediated contacts of small sub-100-nmsized vesicles with the cellular membrane are accompanied by the redistribution of ligands and receptors between the contactfree and contact regions of the vesicle and cellular membranes. Our formalism allows one to scrutinize this effect. In particular, our analysis shows that for physically reasonable values of ligand-receptor interaction, the concentrations of ligands and receptors are appreciably higher in the contact region, and the ratios of the specific concentrations in this region may be different compared to those in the contact-free region.

In addition, our formalism makes it possible to calculate the contribution of the ligand-receptor interaction to the change of the free energy of a vesicle as a function of $N_{*} / N, S_{*} / S$ or $h / 2 R$. This contribution, $\Delta F_{\mathrm{lr}}$, is negative, and with increasing $N_{*} / N, S_{*} / S$, or $h / 2 R$ its absolute value increases first linearly then in a weaker fashion. The two other contributions to the change of the free energy, $\Delta E_{\mathrm{b}}$ and $\Delta E_{\mathrm{c}}$, related to the membrane bending energy and deformation of cytoskeleton [Eq. (11)], are positive. In combination, these contributions form a potential energy of interaction of a vesicle with the cellular membrane. 
For orientation, it is instructive to recall that in the analytically tractable situations when a vesicle remains to be spherically shaped the latter two contributions can be represented as $[22,47]$

$$
\begin{gathered}
\Delta E_{\mathrm{b}}=4 \pi \kappa h / R, \\
\Delta E_{\mathrm{c}}= \begin{cases}0 & \text { for } h<h_{\circ}, \\
\beta\left(h-h_{\circ}\right)^{4} & \text { for } h>h_{\circ},\end{cases}
\end{gathered}
$$

where $\kappa$ is the bending modulus of the cellular membrane, $h_{\circ}$ is the engulfment depth corresponding to the beginning of deformation of actin filaments, and $\beta$ is the related coefficient. If $h$ is appreciable ( $\simeq R$ or larger), the dependence of $\Delta E_{\mathrm{b}}$ and $\Delta E_{\mathrm{c}}$ on $h$ is stronger than that of $\Delta F_{\mathrm{lr}}$. Thus, with increasing $h$, the potential of interaction of a vesicle with the cellular membrane first decreases and then reaches a minimum and starts to increase as it was phenomenologically discussed earlier in the context of virus entry by endocytosis $[22,47]$ (without detalization of $\Delta F_{\text {lr }}$ ).

Concerning the membrane bending, we note that according to Eq. (23), the bending energy per site is $4 \pi \kappa a / R^{2}$, where $a$ is the site area. The scale of $\kappa$ is often considered to be $25 k_{\mathrm{B}} T$ [20] or higher [48], while the scale of $a / R^{2}$ is above $1 / 100$. Thus, the scale of the bending energy per site is $4 k_{\mathrm{B}} T$ or higher. According to Eq. (9), on the other hand, the effective ligand-receptor interaction energy per site is predicted to be $k_{\mathrm{B}} T \theta \Theta \exp \left(U / k_{\mathrm{B}} T\right)$ [20]. This expression was derived assuming $\theta \Theta \exp \left(U / k_{\mathrm{B}} T\right) \ll 1$, and accordingly it is applicable if the effective ligand-receptor interaction energy per site is much smaller than $k_{\mathrm{B}} T$. In this limit, the ligand-receptor interaction is much weaker than the bending energy and can hardly be sufficient for penetration of a filled vesicle through the cellular membrane.

Finally, it is of interest to discuss briefly our results in the context of vesicle- or lipid-nanoparticle-mediated drug (e.g., RNA) delivery. By analogy with viral endocytosis, this process may mechanistically be endocytotic, with wrapping of a vesicle by the host membrane, or fusogenic, with fusion of the vesicle membrane with the host membrane. The related models (see, e.g., Refs. [21,24]) have primarily been focused on the identification of the optimal nanoparticle-size range, $\simeq 50-100 \mathrm{~nm}$, for the entry. For smaller sizes, the activation energy for the penetration through the membrane is too large because the adhesion is not able to compensate the bending energy. For larger particles, the process is too slow due to receptor $2 \mathrm{D}$ diffusion and/or appreciable energy of the cytoskeleton deformation (the latter is discussed in Ref. [22]).
Our analysis indicates that the ligand concentration and composition can be used as efficient factors for optimization of the delivery.

For rapid entry, the population of ligands in a vesicle (or lipid nanoparticle) should be appreciable (comparable with its maximum value). Otherwise, the ligands will be located primarily in the contact area already when this area is small compared the whole vesicle area, the minimum of the potential energy will be reached already at this stage, and accordingly the subsequent appreciable wrapping of a vesicle will be highly unfavorable due to appreciable increase of the free energy related to the membrane bending and deformation of cytoskeleton. In addition, the bending of the vesicle membrane should not be too low. Otherwise, a vesicle contacting the cellular membrane can easily be appreciably flattened (to increase the contact area) so the contact interface remains to be nearly flat even if its radius is appreciable, and accordingly the subsequent appreciable wrapping of a vesicle will also be unfavorable (this effect was earlier discussed in [26]; the ligand redistribution was not treated there). Concerning this condition, one can notice that the bending can be prevented at least partly by the content inside a vesicle. For the fusogenic pathway, the contact area may be small, the population of ligands may be small as well, but it requires the presence of special molecules facilitating the pore formation.

At first sight, rapid entry is desirable. In reality, however, lipid nanoparticles used as carriers are often rapidly trapped inside endosomes, and the delivery of the substance, e.g., RNA, to the cytoplasm is frequently limited by its release from a nanoparticle, which is interfering with the release of a nanoparticle from an endosome [49,50]. Under such circumstances, it might sometimes be favorable to make the entry slower to optimize the whole process of the RNA delivery and function. One of the suitable ways here is to modify the concentration and composition of ligands.

Concerning applications, we may add that vesicles or lipid nanoparticle represent only two examples of biologically inspired candidates for drug delivery. Other similar relevant examples are nanomicelles [51,52] and bicelles [53,54]. Among less conventional candidates, one may mention, e.g., pollen [55].

\section{ACKNOWLEDGMENTS}

This work was supported by (i) Swedish Foundation for Strategic Research (Project No. IRC15-0065) and (ii) Russian Academy of Sciences and Federal Agency for Scientific Organizations (Project No. 0303-2016-0001). The author thanks Fredrik Höök for useful discussions.
[1] M. Yanez-Mo et al., Biological properties of extracellular vesicles and their physiological functions, J. Extracell. Vesicl. 4, 27066 (2015).

[2] E. Barrow, A. V. Nicola, and J. Liu, Multiscale perspectives of virus entry via endocytosis, Virology J. 10, 177 (2013).

[3] L. M. Kranz et al., Systemic RNA delivery to dendritic cells exploits antiviral defense for cancer immunotherapy, Nature 534, 396 (2016).
[4] M. W. Tibbitt, J. E. Dahlman, and R. Langer, Emerging frontiers in drug delivery, J. Am. Chem. Soc. 138, 704 (2016).

[5] M. A. Oberli et al., Lipid nanoparticle assisted mRNA delivery for potent cancer immunotherapy, Nano Lett. 17, 1326 (2017).

[6] M. Mammen, S.-K. Choi, and G. M. Whitesides, Polyvalent interactions in biological systems: implications for design and use of multivalent ligands and inhibitors, Angew. Chem., Int. Ed. Engl. 37, 2754 (1998). 
[7] S. Block, V. P. Zhdanov, and F. Höök, Quantification of multivalent interactions by tracking single biological nanoparticle mobility on a lipid membrane, Nano Lett. 16, 4382 (2016).

[8] S. Block, B. J. Fast, A. Lundgren, V. P. Zhdanov, and F. Höök, Two-dimensional flow nanometry of biological nanoparticles for accurate determination of their size and emission intensity, Nat. Commun. 7, 12956 (2016).

[9] C. Zhu, Kinetics and mechanics of cell adhesion, J. Biomech. 33, 23 (2000).

[10] T. R. Weikl and R. Lipowsky, Membrane adhesion and domain formation, in Advances in Planar Lipid Bilayers and Liposomes, edited by A. L. Liu (Elsevier, Amsterdam, 2006), Vol. 5, pp. 63-127.

[11] T. R. Weikl, M. Asfaw, H. Krobath, B. Rozycki, and R. Lipowsky, Adhesion of membranes via receptor-ligand complexes: Domain formation, binding cooperativity, and active processes, Soft Matter 5, 3213 (2009).

[12] J. A. Sanz-Herrera and E. Reina-Romo, Cell-biomaterial mechanical interaction in the framework of tissue engineering: insights, computational modeling and perspectives, Int. J. Mol. Sci. 12, 8217 (2011).

[13] T. R. Weikl, J. Hu, G.-K. Xu, and R. Lipowsky, Binding equilibrium and kinetics of membrane-anchored receptors and ligands in cell adhesion: Insights from computational model systems and theory, Cell Adhes. Migr. 10, 576 (2016).

[14] B. Rozycki, R. Lipowsky, and T. R. Weikl, Segregation of receptorligand complexes in cell adhesion zones: Phase diagrams and the role of thermal membrane roughness, New J. Phys. 12, 095003 (2010).

[15] T. Bihr, U. Seifert, and A.-S. Smith, Multiscale approaches to protein-mediated interactions between membranes-relating microscopic and macroscopic dynamics in radially growing adhesions, New J. Phys. 17, 083016 (2015).

[16] S. Ostvar and B. D. Wood, Multiscale model describing bacterial adhesion and detachment, Langmuir 32, 5213 (2016).

[17] S. Sircar, G. Nguyen, A. Kotousov, and A. J. Roberts, Ligandmediated adhesive mechanics of two static, deformed spheres, Eur. Phys. J. E 39, 95 (2016).

[18] A. Bunker, A. Magarkar, and T. Viitala, Rational design of liposomal drug delivery systems, a review: Combined experimental and computational studies of lipid membranes, liposomes and their PEGylation, Biochim. Biophys. Acta 1858, 2334 (2016).

[19] T. R. Weikl and R. Lipowsky, Adhesion-induced phase behavior of multicomponent membranes, Phys. Rev. E 64, 011903 (2001).

[20] H. Krobath, B. Rozycki, R. Lipowsky, and T. R. Weikl, Binding cooperativity of membrane adhesion receptors, Soft Matter 5, 3354 (2009).

[21] H. Gao, W. Shi, and L. B. Freund, Mechanics of receptormediated endocytosis, Proc. Natl. Acad. Sci. USA 102, 9469 (2005).

[22] V. P. Zhdanov, Kinetics of virus entry by endocytosis, Phys. Rev. E 91, 042715 (2015).

[23] A. Banerjee, A. Berezhkovskii, and R. Nossal, Kinetics of cellular uptake of viruses and nanoparticles via clathrin-mediated endocytosis, Phys. Biol. 13, 016005 (2016).

[24] S. Zhang, J. Li, G. Lykotrafitis, G. Bao, and S. Suresh, Sizedependent endocytosis of nanoparticles, Adv. Mater. 21, 419 (2009).
[25] H. Yuan and S. Zhang, Effects of particle size and ligand density on the kinetics of receptor-mediated endocytosis of nanoparticles, Appl. Phys. Lett. 96, 033704 (2010).

[26] X. Yi, X. Shi, and H. Gao, Cellular Uptake of Elastic Nanoparticles, Phys. Rev. Lett. 107, 098101 (2011).

[27] X. Li, Size and shape effects on receptor-mediated endocytosis of nanoparticles, J. Appl. Phys. 111, 024702 (2012).

[28] A. Chaudhuri, G. Battaglia, and R. Golestanian, The effect of interactions on the cellular uptake of nanoparticles, Phys. Biol. 8, 046002 (2011).

[29] D. Gonzalez-Rodriguez and A. I. Barakat, Dynamics of receptor-mediated nanoparticle internalization into endothelial cells, PLoS ONE 10, e0122097 (2015).

[30] S. Angioletti-Uberti, Exploiting Receptor Competition to Enhance Nanoparticle Binding Selectivity, Phys. Rev. Lett. 118, 068001 (2017).

[31] X. Yi and H. Gao, Kinetics of receptor-mediated endocytosis of elastic nanoparticles, Nanoscale 9, 454 (2017).

[32] H.-M. Ding and Y.-Q. Ma, Role of physicochemical properties of coating ligands in receptor-mediated endocytosis of nanoparticles, Biomaterials 33, 5798 (2012).

[33] V. Schubertova, F. J. Martinez-Veracoechea, and R. Vacha, Influence of ligand distribution on uptake efficiency, Soft Matter 11, 2726 (2015).

[34] H.-M. Ding and Y.-Q. Ma, Design strategy of surface decoration for efficient delivery of nanoparticles by computer simulation, Sci. Rep. 6, 26783 (2016).

[35] J. Liang, P. Chen, B. Dong, Z. Huang, K. Zhao, and L.-T. Yan, Ligand-receptor interaction-mediated transmembrane transport of dendrimer-like soft nanoparticles: Mechanisms and complicated diffusive dynamics, Biomacromol. 17, 1834 (2016).

[36] Z. Guan, L. Wang, and J. Lin, Interaction pathways between plasma membrane and block copolymer micelles, Biomacromol. 18, 797 (2017).

[37] Y. Li, B. Yuan, K. Yang, X. Zhang, B. Yan, and D. Cao, Counterintuitive cooperative endocytosis of like-charged nanoparticles in cellular internalization: Computer simulation andexperiment, Nanotechnology 28, 085102 (2017).

[38] A.-S. Smith and U. Seifert, Effective adhesion strength of specifically bound vesicles, Phys. Rev. E 71, 061902 (2005).

[39] L. Simonsson and F. Höök, Formation and diffusivity characterization of supported lipid bilayers with complex lipid compositions, Langmuir 28, 10528 (2012).

[40] S. R. Tabaei, J.-H. Choi, G. H. Zan, V. P. Zhdanov, and N.-J. Cho, Solvent-assisted lipid bilayer formation on silicon dioxide and gold, Langmuir 30, 10363 (2014).

[41] J. R. Houser, D. J. Busch, D. R. Bell, B. Li, P. Rena, and J. C. Stachowiak, The impact of physiological crowding on the diffusivity of membrane bound proteins, Soft Matter 12, 2127 (2016).

[42] L. D. Landau and E. M. Lifshitz, Statistical Physics (Elsevier, Amsterdam, 1980).

[43] T. L. Hill, Statistical Mechanics (McGraw-Hill, New York, 1956).

[44] V. P. Zhdanov, Elementary Physicochemical Processes on Solid Surfaces (Plenum, New York, 1991).

[45] C. Guardiani, R. Livi, and F. Cecconi, Coarse grained modeling and approaches to protein folding, Curr. Bioinform. 5, 217 (2010). 
[46] V. P. Zhdanov and B. Kasemo, Monte Carlo simulations of the kinetics of protein adsorption, Surf. Rev. Lett. 5, 615 (1998).

[47] V. P. Zhdanov, Physical aspects of the initial phase of endocytosis, Phys. Rev. E 88, 064701 (2013).

[48] J. A. Jackman et al., Quantitative profiling of nanoscale liposome deformation by a localized surface plasmon resonance sensor, Anal. Chem. 89, 1102 (2017).

[49] R. L. Juliano, The delivery of therapeutic oligonucleotides, Nucl. Acids Res. 44, 6518 (2016).

[50] S. F. Dowdy, Overcoming cellular barriers for RNA therapeutics, Nat. Biotechnol. 35, 222 (2017).

[51] J. Zhou et al., pH-sensitive nanomicelles for high-efficiency siRNA delivery in vitro and in vivo: An insight into the design of polycations with robust cytosolic release, Nano Lett. 16, 6916 (2016).

[52] P. E. Saw, M. Yu, M. Choi, E. Lee, S. Jon, and O. C. Farokhzad, Hyper-cell-permeable micelles as a drug delivery carrier for effective cancer therapy, Biomaterials 123, 118 (2017).

[53] G. Rodriguez et al., Bicelles: New lipid nanosystems for dermatological applications, J. Biomed. Nanotechnol. 11, 282 (2015).

[54] Y. Liu, Y. Xia, A. T. Rad, W. Aresh, and M.-P. Nieh, Stable discoidal bicelles: A platform of lipid nanocarriers for cellular delivery, Meth. Molec. Biol. 1522, 273 (2017).

[55] R. C. Mundargi et al., Natural sunflower pollen as a drug delivery vehicle, Small 12, 1167 (2016). 